\title{
COMBINED ALEXA-488 AND NANOGOLD ANTIBODY PROBES
}

\author{
W. Liu, J. F. Hainfeld, and R. D. Powell
}

Nanoprobes, Incorporated, 95 Horseblock Rd., Yaphank NY 11980

Using sequential cross-linking steps, the $1.4 \mathrm{~nm}$ Nanogold gold cluster label and a fluorescent tag may both be attached to an antibody or other targeting agent to yield a probe which may be used for 'simultaneous' or correlative fluorescence and electron microscopic labeling $[1,2]$. Preparation and use of combined Nanogold/Fluorescein and Nanogold/Cy3 [3] probes have been described. However, fluorescein is not ideal for all fluorescence experiments. It is subject to photobleaching, and can be affected by $\mathrm{pH}$. It is also relatively hydrophobic, a potential cause of non-specific binding in some specimens. The development of new fluorophores such as the ALEXA dyes [4] with more intense fluorescence, higher solubility, improved stability and lower self-quenching affords opportunities to prepare combined fluorescent and gold probes with improved staining properties.

A combined ALEXA 488 and Nanogold probe was prepared by the reduction of F(ab') 2 Goat anti-Mouse IgG to Fab' fragments with mercaptoethylamine hydrochloride (MEA), which selectively reduce the $\mathrm{F}(\mathrm{ab})_{2}$ hinge disulfide bonds, with $5 \mathrm{~mm}$ EDTA present to prevent reoxidation. Fab' fragments were isolated by gel filtration (coarse gel: GH25, Millipore) then labeled selectively at the reduced hinge thiol using Monomaleimido-Nanogold [5]. The conjugate was isolated by gel filtration (Superose-12, Pharmacia), then reacted with excess ALEXA Fluor 488 amine labeling kit (Molecular Probes); dual-labeled conjugates were isolated by gel filtration (Superose-12). In this way, the fluorescent group was positioned sufficiently far from the Nanogold that quenching through fluorescence resonance energy transfer [1] was minimized. UV/visible spectroscopy indicated incorporation of 2-3 ALEXA molecules per Fab'; preliminary fluorescence measurements suggested that fluorescence emission intensities relative to the unconjugated ALEXA dye were more than twice those of combined fluorescein and Nanogold conjugates relative to unconjugated fluorescein.

The combined ALEXA 488 and Nanogold conjugate was used to stain cytokeratin in paraffin-embedded human prostate carcinoma test slides; bright, clear fluorescence labeling was found, as shown in Figures 1 and 2. Localization of the gold was demonstrated by brightfield microscopy after silver enhancement (Figure 3); specificity was confirmed by the absence of labeling when the primary antibody was omitted (Figure 4). Further experiments are in progress to characterize and optimize the performance of this probe, and to prepare combined fluorescent and Nanogold probes using other ALEXA dyes.

\section{References:}

[1] R. D. Powell, C. M. R. Halsey, and J. F. Hainfeld: Microsc. Res. Tech., 42 (1998) 2.

[2] T. Takizawa, K. Suzuki, and J. M. Robinson: J. Histochem. Cytochem., 46 (1998) 1097.

[3] R. D. Powell, V. N. Joshi, C. M. R. Halsey, J. F. Hainfeld, G. W. Hacker, C. HauserKronberger, W. H. Muss, and P. M. Takvorian,: Microsc. Microanal., (Suppl. 2: Proceedings), 6 (1999) 478.

[4] N. Panchuk-Voloshina, R. P. Haugland, J. Bishop-Stewart, M. K. Bhalgat, P. J. Millard, F. Mao, and W. Y. Leung: J. Histochem. Cytochem., 47 (1999) 1179.

[5] J. F. Hainfeld and F. R. Furuya: J. Histochem. Cytochem., 40 (1992) 177. 
[6] The authors thank Molecular Probes for providing ALEXA Fluor 488 amine labeling reagent. This project was partly supported by SBIR grant 1R43 GM62100-01 from the National Institute of General Medical Sciences (National Institutes of Health).
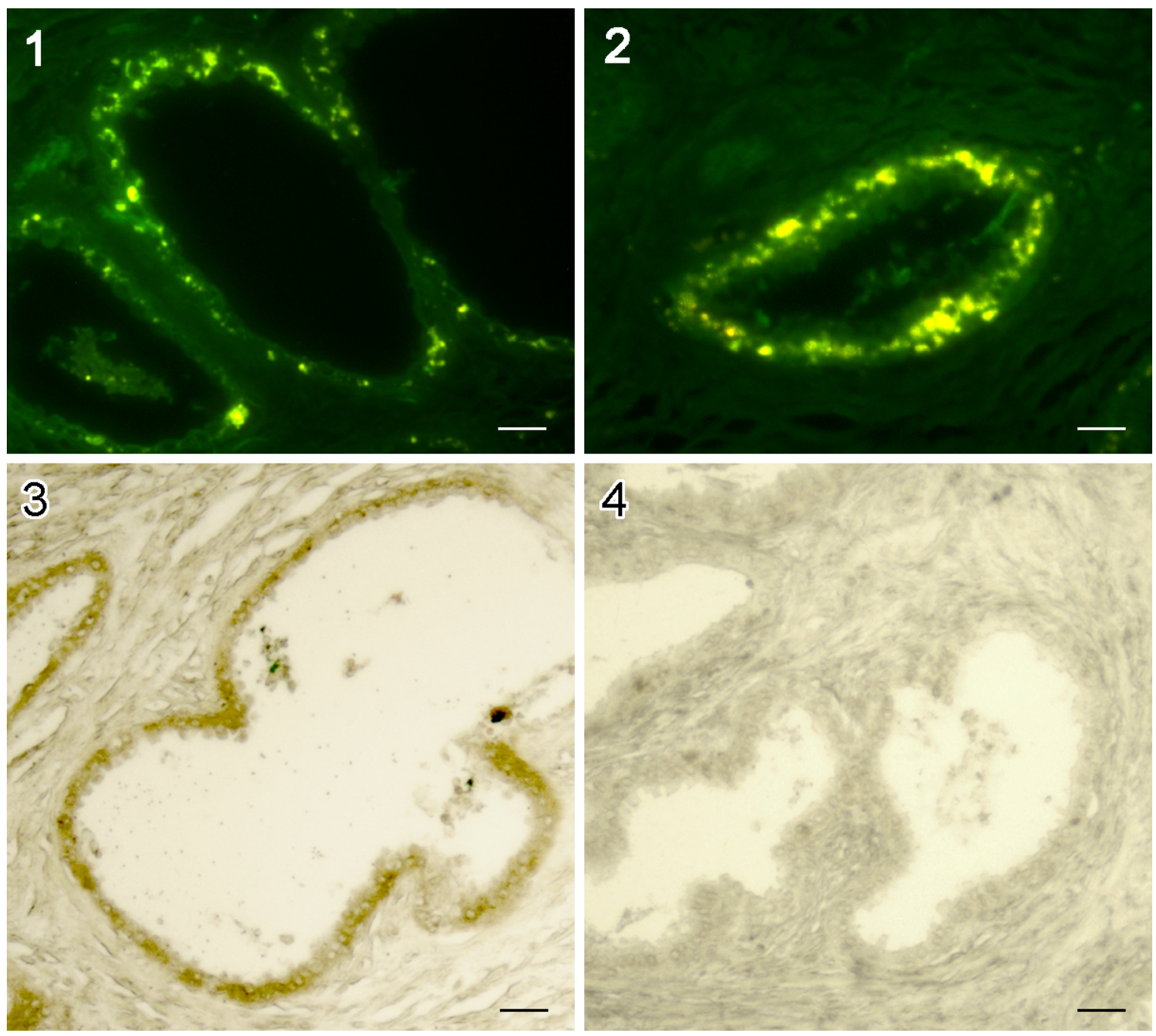

Figure 1: Fluorescence micrograph of paraffin section of human prostate carcinoma (Dako Cytokeratins Human Adeno CA prostate slide) stained for cytokeratin using monoclonal primary antibody (AE1/AE3, Dako: $1: 50$ dilution in Tris-buffered saline), and combined ALEXA488/Nanogold-Fab' goat anti-Mouse IgG (final concentration $28 \mu \mathrm{g} / \mathrm{mL}$ ) secondary $($ bar $=100 \mu$ ).

Figure 2: Higher magnification view of same specimen as Figure $1($ bar $=25 \mu)$.

Figure 3: Brightfield light micrograph of paraffin section of human prostate carcinoma (Dako Cytokeratins Human Adeno CA prostate slide) stained for cytokeratin as in Figure 1, followed by silver enhancement with LI Silver (Nanoprobes) for 15 minutes $(\mathrm{bar}=25 \mu)$.

Figure 4: Negative control: paraffin section of human prostate carcinoma (Dako Cytokeratins Human Adeno CA prostate slide) stained and silver enhanced as in Figure 3, except that primary antibody was omitted $($ bar $=25 \mu)$. 